\title{
Immunological aspects of ovarian malignancy
}

\author{
Agrimaldo Martins-Filho ${ }^{1}$, Millena Prata Jammal ${ }^{1}$, Eddie Fernando Candido Murta ${ }^{1}$, \\ Rosekeila Simões Nomelini ${ }^{1, *}$ () \\ ${ }^{1}$ Research Institute of Oncology (IPON)/Department of Gynecology and Obstetrics, Federal University of Triângulo Mineiro, 38025-440 Uberaba-MG, \\ Brazil \\ *Correspondence: rosekeila@terra.com.br; rosekeila.nomelini@pesquisador.cnpq.br (Rosekeila Simões Nomelini) \\ Academic Editor: Michael H. Dahan \\ Submitted: 22 June 2021 Revised: 22 July 2021 Accepted: 9 August 2021 Published: 8 February 2022
}

\begin{abstract}
Objective: Evidence shows that the host's immune system interacts with tumor development and may be responsible for delaying tumor growth or even for its progression. Chronic inflammation has been identified as a key factor in the pathogenesis of ovarian cancer. The objective of this study is to carry out a comprehensive review of immunological aspects of ovarian cancer. Mechanism: A search of the following keywords was conducted in the PubMed database: "ovarian cancer", "prognostic factors", "immunotherapy", "immune system". The articles identified were published between 1992 and 2021. Findings in brief: A total of 75 articles were selected for further examination. Conclusions: The understanding of tumor immune escape mechanisms will improve immunotherapy treatments. This will allow personalization or treatment not only in accordance with the biology of the tumor, but also with the characteristics of the tumor microenvironment, expanding the benefit of immunotherapy and providing more effective responses.
\end{abstract}

Keywords: Ovarian cancer; Prognostic factors; Immunotherapy; Immune system

\section{Introduction}

Ovarian cancer is the seventh most common cancer in women and the third most common cancer worldwide, after breast and cervical cancer. It is the fifth most frequent cause of death among gynecological malignancies [1]. According to GLOBOCAN [2], an estimated 313,959 new cases will be diagnosed worldwide and approximately 207,252 women will die as a result of the disease. The American Cancer Society estimates that about 21,410 women in the United States will be newly diagnosed with ovarian cancer in 2021 , and that about 13,770 of these women will die of the disease [3].

Ovarian cancer encompasses a collection of neoplasms with distinct clinicopathological and molecular characteristics. Its etiology appears to be multifactorial, including reproductive, familial, and individual factors [4]. About $90 \%$ of ovarian cancers are sporadic, with no recognized risk factor. About $10 \%$ have a genetic or familial component. Because its development is asymptomatic, ovarian cancer is often diagnosed late, in the presence of extensive peritoneal dissemination and distant metastasis [5]. About $70 \%$ of cases are diagnosed in the advanced stage of the disease [6], making ovarian cancer highly lethal and rapidly progressive.

The standard treatment for ovarian cancer is cytoreductive surgery followed by combined platinum/taxane chemotherapy. For patients diagnosed with stage IA disease, the chance of a cure can reach $90 \%$; for those diagnosed with stage II disease, the cure potential reaches $70 \%$. The cure rate for ovarian cancer diagnosed in stages III and
IV reaches only $30 \%$. Relapse occurs within 2 years in most cases, and some patients become resistant to chemotherapy, resulting in a 5 -year survival rate of only $45 \%$ at best $[7,8]$. For tumors limited to the pelvis, the 5-year survival rate can reach $60 \%$ [9]. Although ovarian cancer has a variety of subtypes, it is treated as a single disease, which partially explains the lack of successful treatment.

Several studies have been conducted in the attempt to improve the classification of ovarian tumors, opening the horizon for new knowledge about the origins of the different histological types of ovarian cancer and new paths for basic research and clinical studies. Despite many years of research, however, reliable diagnostic markers and other methods enabling early detection and performing adequately for screening and disease management remain lacking. Thus, current studies are aiming to identify new biomarkers with potential for ovarian cancer diagnosis, prognosis, and prediction, as well as new therapeutic targets. This comprehensive review examines immunological aspects of ovarian malignancy, summarizing the main findings from the literature.

\section{Methods}

The PubMed database was searched using the keywords "ovarian cancer", "prognostic factors", "immunotherapy", and "immune system". The criteria for the inclusion of an article in this review were publication in or translation to English or Portuguese; original research, systematic review, or review; and publication in a journal between January 1992 and May 2021. 


\section{Results}

In this literature review, we discuss prognostic factors for ovarian cancer, the role of the immune system in ovarian cancer, cells of the immune system, the role of the stroma in the immunology and prognosis of ovarian cancer, and immunotherapies for this disease. In total, 75 articles were selected for further examination.

\subsection{Prognostic factors}

Ovarian cancer is highly lethal; its overall prognosis has remained poor over the past few decades [10]. Twothirds of affected patients have advanced disease, and the majority of them will experience disease relapse requiring continuous treatment; many of these patients will die of the disease [11].

Prognostic factors defined by correlation with survival generally reflect the extent of the disease (staging), the intrinsic biology of the tumor (type and histological grade), and the patient's ability to tolerate treatment of the disease. In addition to these pre-treatment factors, others that may affect survival include the type of treatment received (e.g., optimal debulking, chemotherapy based on platinum derivatives) and the effects of the therapy on the tumor (e.g., complete response, other) and the patient (e.g., myelosuppression) [12]. Among these prognostic factors, the International Federation of Gynecology and Obstetrics staging is considered to be the most important [4], followed by the presence of residual disease after surgery and the initial histological type and tumor grade. Several groups have evaluated the clinical relevance of different biological variables in tissue and serum samples from patients with ovarian cancer in the effort to identify biomarkers capable of predicting the response to chemotherapy or patient survival. Studies of the roles of individual molecular markers have demonstrated that ovarian carcinogenesis is a complex, multifactorial process associated with abnormalities in several families of genes, which could have predictive or prognostic relevance [13]. Primary cytoreductive surgery followed by adjuvant chemotherapy remains the standard treatment for patients with ovarian cancer. However, the survival rate varies considerably among patients with the same pathological stage of disease receiving the same treatment, which may be attributable to host-related factors, including variations in the inflammatory response [14].

The identification of new biomarkers for the diagnosis and prognosis of ovarian cancer, which ideally also serve as targets for new therapeutic modalities, is urgently needed [15]. Transvaginal ultrasound and cancer antigen 125 (cancer antigen 125 (CA-125)) detection are used commonly to detect ovarian cancer [16]. Over time, the use of a combination of biomarkers (carcinoembryonic antigen, carbohydrate antigen 19-9, and Human Epididymis protein 4) together with CA-125 has emerged, but no biomarker in clinical use for the early detection of ovarian cancer is fully effective due to a lack of sensitivity or specificity [17]. In the future, the identification of new biomarkers will also aid the identification of specific markers for molecular targeted therapy [18].

In recent years, laboratory-quantified systemic inflammatory response markers, such as hypoalbuminemia, hyperfibrinogenemia, the C-reactive protein level, the absolute leukocyte count, the neutrophil/lymphocyte ratio (NLR), and the platelet/lymphocyte ratio (PLR), have been introduced as prognostic factors for various types of cancer, including ovarian cancer [14,19]. The NLR and PLR have recently been shown to be significant predictors of malignancy for solid tumors originating from various tissues; they are also useful in screening for these tumors, as they can be determined using inexpensive and readily available tests [20]. However, the authors reporting on this research indicated the need for further assessment of the additional value of these findings, the establishment of scores, and the determination of the potential predictive value of these markers for gynecological cancers [20]. Sanguinete et al. [21] demonstrated that increased levels of interleukin (IL)6 and IL-8, observed in the more advances stages of ovarian cancer, were associated with better prognoses. Various theories have been proposed to explain the function of the immune system in relation to tumor cells and their respective signaling molecules. IL levels are found in patients with ovarian cancer related to prognostic factors [21-23].

\subsection{The role of the immune system in ovarian cancer}

The pathophysiology of ovarian cancer is complex and remains poorly understood. Its diagnosis is affected greatly by the absence of specific signs and symptoms, the lack of reliable biomarkers, and the anatomical location of the ovaries. Thus, ovarian cancer is often diagnosed in the advanced stage of the disease, rendering its prognosis uncertain [24] and of limited value. Thus, researchers have sought to identify means by which to improve the prognosis of the disease and better guide its therapeutic management [25].

The host immune system interacts with tumor development, and in some cases may be responsible for the delay of tumor growth or progression. Chronic inflammation has been identified as a key factor in the pathogenesis of ovarian cancer [26]. Ovulation is a potentially inflammatory and mutagenic process [27]. Most tumors have complex relationships with their hosts, with the presence of inflammatory cells and mediators in the tumor microenvironment resulting in the production of chemokines, cytokines, and prostaglandins, which recruit inflammatory cells (e.g., neutrophils, mast cells, and macrophages) and stimulate angiogenesis and cell proliferation [28,29]. In addition, tumors can evade immunovigilance, resulting in a certain degree of tolerance by the immune system, and their ability to interfere with immune cell function seems to be the main reason for the failure to control tumor progression [30].

Inflammation influences all stages of cancer develop- 
ment, including tumor initiation, promotion, and progression [26]. Several inflammatory mediators are induced by inflammatory and/or tumor cells and participate in the development of cancer, acting as growth or angiogenic factors. In addition, immune function is compromised by these mediators, causing increases in the levels of leukocytes, neutrophils, platelets, C-reactive protein, and fibrinogen, and reducing the concentrations of lymphocytes and albumin. Cancer-induced thrombocytosis can significantly increase the number of circulating platelets, by mechanisms that remain poorly understood [31]. A multicenter study conducted by Stone et al. [32] showed that thrombocytosis is associated significantly with poor prognosis and directly influences patient survival. In addition, that study revealed that the inflammatory cytokine IL-6 influences thrombocytosis in patients with ovarian cancer by stimulating hepatic thrombopoietin synthesis [32].

Adaptive and innate immune responses, including cytotoxic and antibody secretion processes, are dysfunctional in patients with cancer, which can increase susceptibility to cancer cell growth and invasion and thus to tumor development [33,34]. An escape mechanism that favors the maintenance of ovarian cancer is the triggering of an imbalance in T helper 1 (Th1) and T helper 2 (Th2) responses, with an increase in the latter that results in impairment of the processes that control homeostasis in the tumor microenvironment [35]. The Th1-type immune response stimulates cellular immunity by activating macrophages and dendritic cells and recruiting cluster of differentiation $8+(\mathrm{CD} 8+)$ cytotoxic $\mathrm{T}(\mathrm{Tc})$ cells and natural killer (NK) cells. The Th2type immune response inhibits cell-mediated immunity and favors the humoral immune response. The generation of a Th1- or Th2-type immune response depends on the cytokine balance [36]. Increasing evidence indicates that $\mathrm{T}$ cell activation and the resulting cytokine expression play key roles in the diminution and disruption of inflammatory and antitumor immune responses [37].

The environment in which ovarian carcinoma develops has been described as large and rich in proinflammatory cytokines and chemokines. This tumor produces IL$1 \beta$, IL-6, and IL-12, which in addition to the stimulation of cancer cell growth are responsible for the creation of a microenvironment promoting cell proliferation and tumor growth, thereby directly affecting the severity of inflammation [38]. The unbalanced production of Th1 and Th2 cytokines can favor changes in the secretion of proinflammatory cytokines and consequently aggravate tumor progression [39]. Sanguinete et al. [21] demonstrated that increased IL-6 and IL-8 levels were associated with a worse prognosis of ovarian cancer.

\subsection{Cells of the immune system}

Several elements of the immune response to ovarian cancers have prognostic significance. The presence of different leukocyte populations infiltrating or associated with the tumor has been correlated with better and worse prognoses of the disease, indicating the protective and, at the same time, promoter of the immune system against cancer [40-43].

Studies performed with various tumor tissues have demonstrated strong infiltration of leukocytes into the intratumoral and peritumoral regions. The presence of these cells is related to responses to tumors, due to pre-cancer inflammatory processes or by substances produced by the tumor cells [44]. Their detection in ovarian tumors enables better prediction of clinical outcomes than achieved with the use of other histopathological parameters, indicating that the immunological profile can be useful in conducting alternative forms of treatment, such as immunotherapy $[24,45]$.

\subsubsection{T lymphocytes}

Tumor-infiltrating lymphocytes (TILs) are T cells, B cells, macrophages, and NK cells that have left the vasculature and moved to the tumor stroma or intraepithelium [46]. cluster of differentiation 3+ T lymphocytes (TCD3) + lymphocytes represent the largest leukocyte population detected in ovarian tumors. They are found in tumors (as TILs) and in the ascitic fluid (as tumor-associated lymphocytes). Relative to their absence, the presence of TILs is related to a higher survival rate in patients with stages III and IV ovarian cancer. Optimal cytoreduction also has been observed in most patients with such cells [47].

Levels of $\mathrm{CD} 3+\mathrm{CD} 8+\mathrm{T}$ cells are significantly higher in carcinomas than in benign ovarian epithelial neoplasms, suggesting that the presence of these lymphocytes is related to a better prognosis in terms of the clinical response of ovarian cancer [48]. Regulatory $\mathrm{T}$ (Treg) cells can block the protective effects of tumor-specific T lymphocytes, suppressing their production of interferon- $\gamma$ and IL-2, in patients with ovarian cancer. A high rate of Treg cells has been identified as a predictor of the risk of death and reduced survival in patients with ovarian cancer of all stages [49].

\subsubsection{NK T lymphocytes}

Natural killer T (NK T) lymphocytes have receptors for NK cells and T lymphocytes. In ovarian cancer, as in melanoma and breast, pancreatic, lung, and colorectal cancers, NK T cells have been associated with the inhibition of tumor growth through tumor cell lysis [50]. In patients with ovarian cancer, the levels of NK T cells in the tumor and ascites are significantly higher than that in peripheral blood. The presence of NK T cells in ascites is associated with resistance to chemotherapy with platinum derivatives. Thus, a large number of NK T cells in ascites can be a predictor of platinum sensitivity [25]. 


\subsection{NK lymphocytes}

In patients diagnosed with advanced-stage ovarian cancer, the NK cell level in the peripheral blood is significantly higher than those in the tumor and ascites [51]. Increased numbers of NK cells in ascites have been related to worse clinical outcomes of ovarian cancer and decreased survival [52]. CA-125 can reduce the cytolytic capacity of NK cells by up to $70 \%$. It binds to NK cells, especially to CD16+CD56+ NK cells, which comprise about $90 \%$ of the NK cells present in the peripheral blood [53].

\subsection{Macrophages}

Tumor-associated macrophages (TAMs) affect various aspects of neoplastic tissues, such as their vascularization, growth rate, formation, and stroma dissolution [54]. Macrophages constitute about $51 \%$ of the population of mononuclear cells infiltrated into ovarian carcinomas; they have been observed in all malignant tumors and $57 \%$ of benign tumors, with their average number being significantly higher in the former than in the latter [48].

\subsection{Role of the stroma in ovarian cancer immunology and prognosis}

The survival of tumor cells that metastasize from primary tumors to secondary sites depends on the stromal microenvironment. This metastatic growth occurs primarily with the formation of a pre-metastatic niche before the macroscopic invasion of tumor cells. Exosomes, which are small extracellular vesicles that prepare a distant tumor microenvironment for metastatic invasion, are released from a primary ovarian tumor and regulate communication among tumor cells in the normal stroma, cancer-associated fibroblasts (CAFs), and local immune cells in the tumor microenvironment to support the viability of tumor cells and metastatic dissemination. Functionally, they reprogram stromal cells in the pre-metastatic niche, including CAFs and pericytes. Exosomes also interact with TAMs in the metastatic microenvironment, which are responsible for tumor growth, invasion, angiogenesis, and general metastasis [55].

TAMs are capable of producing matrix metalloproteinases that can be pro- and anti-angiogenic molecules [56]. TAMs of the M1 and M2 types are responsible for the suppression and promotion of cancer progression, respectively [57].

In the tumor microenvironment, the interaction between endothelial cells and pericytes causes angiogenic support of the tumor [58].

CAFs are groups of cells with a reactive common fibroblast phenotype that are induced by cancer cells. This dynamics between CAFs and tumor cells stimulate cancer cells to produce factors that support and activate CAFs, resulting in the promotion of angiogenic events, cellular proliferation, remodeling of the extracellular matrix, and tumor progression [59]. The resulting changes in stromal com- ponents further exacerbate the tumorigenic microenvironment and facilitate oncogenic transformation, tissue invasion, and metastasis during cancer onset and progression [60].

\subsection{Immunotherapies}

Cancer immunotherapy involves the use of different approaches to improve the ability of an individual's immune system to eliminate tumor cells [61]. Current immunotherapy types can be classified as active and passive. Active immunotherapy consists of the stimulation of the immune response against tumor antigens. It can be nonspecific, involving the administration of substances that stimulate and restore the immune system, or specific, i.e., related to tumor vaccines. Active specific immunotherapy can be autologous, performed with vaccines and serums produced from the patient's cell culture, or heterologous, in which these substances are produced from the cells of another patient with a similar neoplasm. Passive immunotherapy consists of the administration of anti-tumor antibodies or effector cells against a certain type of tumor [62].

Trials designed to improve our understanding of the role of active immunotherapy in the treatment of ovarian cancer have been based on the premise that activation of the immune system, which would trigger a more robust immune response with the ability to attack tumor cells and induce apoptosis, would lead to increased tumor clearance. Five main active approaches to immune system sensitization to anti-tumor vaccines are currently used. The first approach is the administration of mesothelin, a protein overexpressed in many ovarian cancers; although clinical studies have shown that patients tolerate this treatment well, no conclusion regarding its effectiveness in the treatment of ovarian cancer has been drawn [63]. The second approach is the administration of a vaccine targeting the New York esophageal squamous cell carcinoma 1 (NY-ESO-1) protein. Differential expression of this antigen has been demonstrated in many ovarian tumors compared with that in normal tissues, and has the function of inducing CD4 and CD8. As a result, patients have improved survival after NY-ESO-1 vaccination [64]. The third approach is the administration of a vaccine containing the tumor suppressor gene p53. Ovarian cancer often expresses a mutant form of p53 early in its development, making it a potential target for immunotherapy. Although evidence supports the safety of this vaccine, its effectiveness has not been determined [65]. The fourth approach is the administration of a vaccine that targets the (Wilms' tumor 1) WT1 and Human Epidermal growth factor Receptor 2 (HER2) antigens. These antigens are particularly attractive as targets due to the frequency with which they are found in ovarian cancer and to the association of HER2 expression with the most aggressive phenotype of the tumor. Vaccines with both antigens have shown promise, but data on their effectiveness from a large prospective randomized controlled trial are lacking 
[66]. The fifth approach is the administration of vaccines based on dendritic cells. Preclinical studies have shown that dendritic cells exposed to ovarian tumor lysates activate $\mathrm{T}$ cells [67], suggesting that therapy with dendritic cells improves tumor-related immune responses. Data on the effectiveness of this treatment from large robust clinical trials are not yet available [68].

Immunological checkpoints constitute a multitude of inhibitory pathways connected to the immune system. Their function is to maintain self-tolerance and the regulation of the timing and amplitude of physiological immune responses in peripheral tissues, to decrease collateral damage to tissue after a response to pathogens. The action of checkpoints in the immune system begins with ligandreceptor interaction, which can be blocked easily with antibodies or modulated with recombinant forms of ligands or receptors [69]. Checkpoint blocking is of great interest in cancer research and is a very promising approach to the improvement of anti-tumor immunity in patients with ovarian cancer. Molecules such as programmed cell death protein 1 (PD-1), cytotoxic antigen associated with the T4 lymphocyte (CTLA-4), lymphocyte activation gene-3, T-cell immunoglobulin, and mucin-3 protein have been targeted in studies of immunological checkpoints [70]. In the context of clinical immunotherapy for cancer, the two most-studied checkpoint-blocking receptors are CTLA-4 (CD152) and PD-1 (CD279); monoclonal antibodies targeting CTLA-4 (binding to CD80/86) and PD-1 or its ligand (PD-L1) have been investigated in several clinical trials [69].

The US Food and Drug Administration has approved six checkpoint inhibitors: ipilimumab (a CTLA-4 antibody); pembrolizumab and nivolumab (PD-1 antibodies); and avelumab, atezolizumab, and durvalumab (PD-L1 antibodies) [71]. Although anti-CTLA-4 and anti-PD-1/PDL1 antibodies increase the anti-tumor immune response by increasing the number of $\mathrm{T}$ cells that infiltrate the tumor and restoring the effector function of Tc cells, their mechanisms of action differ [69].

CTLA-4 (CD152) is present specifically on T cells and shares a set of ligands with the CD28 costimulatory receptor. CD28 and CTLA-4 share two equal ligands: B7.1 (CD80) and B7.2 (CD86). Although disputes occur between these receptors regarding binding to B7.1 and B7.2, CTLA-4 has much greater affinity for both ligands, thus providing for competitive inhibition [69]. Antibodies against CTLA-4 block its ligand binding, thereby increasing CD28-mediated costimulation. CTLA-4 blockade enhances the proliferation and activation of tumor-specific CD8+ T cells [72] and promotes an increase in immune responses that are dependent on Th cells, increasing their suppressive function. The specific CTLA-4 blockade of Treg cells significantly inhibits the ability to regulate autoimmune and anti-tumor immunity [69].

PD-1 is expressed on T cells and inhibits T-cell activity in the effector phase [73]. After activation by its lig- ands PD-L1 (B7-H1 and CD274) and PD-L2 (B7-CD and CD273), PD-1 recruits the inhibitory phosphatase SHP-2, which rapidly dephosphorylates CD28 and inactivates its costimulatory signaling [74]. Of the two PD-1 ligands, PD$\mathrm{L} 1$ is considered to be the most relevant in the tumor microenvironment due to its expression in tumor, stromal, and immunological cells [75]. Antibodies against PD-1 and PDL1 restore the function of tumor-infiltrating $T$ cells, favoring tumor regression [76].

Antibodies targeting PD-1, PD-L1, and CTLA-4 have been tested in patients with recurrent ovarian cancer. In general, response rates range from $5.9 \%$ to $15 \%$ [77]. Anti-CTLA-4 activity yielded greater toxicity and lower response rates than did assays with monoclonal anti-PD1/PD-L1 antibodies [78].

The main barrier to the success of immunotherapy for patients with ovarian cancer remains the immunosuppressive tumor microenvironment. Checkpoint blockade alone may not be sufficient. Although immunotherapy may generate a large number of tumor-specific $\mathrm{T}$ cells, these cells do not always destroy tumor targets easily in vivo, meaning that additional treatment pathways should be studied to improve the effectiveness of the antitumor immune response [79].

The combined use of immunological checkpoint blockers with other immunological inhibitors, such as poly(ADP-ribose) polymerase (PARP) and vascular endothelial growth factor (VEGF) inhibitors, has resulted in progress in immunotherapy for ovarian cancer. PARP inhibitors are used in the treatment of advanced epithelial ovarian cancers with $B R C A$ 1-2 gene mutation. They block base excision repair in cancer cells lacking homologous recombination due to BRCA mutation, resulting in the activation of the synthetic lethality mechanism, with the loss of DNA repair and cell death [80]. The combined use of PARPi olaparib and anti-PD-L1 durvalumab showed modest clinical activity in recurrent ovarian cancer [81]. Vascular endothelial growth factor (VEGF) is known mainly as a mediator of angiogenesis and immune suppression in the tumor microenvironment. Inhibitors targeting VEGF or its receptors reduce the numbers of Treg cells and myeloidderived suppressor cells and increase the infiltration of effector T cells [82].

Checkpoint blockers have been combined with chemotherapy to enhance the anti-tumor immune response, induce direct cytotoxic effects on tumor cells and immunogenic cell death, increase antigen presentation, and alter the inflammatory tumor medium [83]. Five phase III randomized controlled trials examining the addition of PD-L1 blockade to chemotherapy are currently underway; avelumab is being used in two trials and atezolizumab is being used in three trials. The use of durvalumab and a PD-1 inhibitor in combination with chemotherapy is being tested in several phase I/II clinical trials. Based on published results, combined treatment with PD-1/PD-L1 inhibitors and 
chemotherapy is potentially superior to chemotherapy alone [84].

Adoptive cell therapy (ACT) against ovarian tumors is also being studied. Adoptive immunotherapy is emerging as an active treatment for cancers that are less responsive or resistant to treatment with checkpoint blockers. This approach is based on the infusion of autologous or allogeneic immune effectors that destroy tumor cells. One of the most promising approaches for the treatment of solid tumors involves tumor-specific Tc cells isolated from the tumor or peripheral blood, which are expanded ex vivo and then infused after lympho-depleting chemotherapy. Two main forms of ACT are currently being used in cancer therapy: TIL isolation and expansion, and the use of genetically engineered $\mathrm{T}$ cells that express a specific tumor antigen receptor or chimeric antigen receptor [83].

\section{Conclusions and future perspectives}

Ovarian cancer is associated strongly with inflammation, and, in most cases, has a complex relationship with the host, with the presence of inflammatory cells and inflammatory mediators in the tumor microenvironment resulting in increased angiogenesis and cell proliferation. The ability to interfere with immune cell function seems to be the main reason for the failure to control the progression of cancer; the shift in the immune response from Th1 to Th2 cells results in impairment of the processes that control homeostasis, particularly in the tumor stroma. Several clinical trials examining immunotherapy for ovarian cancer are underway. They will help to define the best role for immunotherapy in the treatment of ovarian cancer and prevention of its recurrence. A better understanding of the tumor's immune escape mechanisms will enable the development of more sophisticated immunotherapeutic approaches, including treatment personalization according to tumor biology and the characteristics of the tumor microenvironment. Thus, immunotherapy can lead to long-lasting and effective responses. The identification of new biomarkers is also needed to trigger a maximum anti-tumor immune response. The data presented here show that significant advances are being made in the implementation of immunotherapy for the management of ovarian cancer, which will play an important role in the treatment and prevention of this disease in the future.

\section{Author contributions}

RSN and EFCM designed the research study. AMF and MPJ performed the research. AMF and MPJ wrote the manuscript. All authors contributed to editorial changes in the manuscript. All authors read and approved the final manuscript.

\section{Ethics approval and consent to participate}

Not applicable.

\section{Acknowledgment}

We wish to acknowledge the CNPq (Conselho Nacional de Desenvolvimento Científico e Tecnológico), FUNEPU (Fundação de Ensino e Pesquisa de Uberaba), FAPEMIG (Fundação de Amparo à Pesquisa do Estado de Minas Gerais), and CAPES (Coordenação de Aperfeiçoamento de Pessoal de Nível Superior - Brasil) for research support.

\section{Funding}

This research received no external funding.

\section{Conflict of interest}

The authors declare no conflict of interest. EFCM is our Editor Board, given his role as Editor Board, had no involvement in the peer-review of this article and has no access to information regarding its peer-review.

\section{References}

[1] Ottevanger PB. Ovarian cancer stem cells more questions than answers. Seminars in Cancer Biology. 2017; 44: 67-71.

[2] Sung H, Ferlay J, Siegel RL, Laversanne M, Soerjomataram I, Jemal A, et al. Global Cancer Statistics 2020: GLOBOCAN Estimates of Incidence and Mortality Worldwide for 36 Cancers in 185 Countries. CA: A Cancer Journal for Clinicians. 2021; 71: 209-249.

[3] Siegel RL, Miller KD, Jemal A. Cancer statistics, 2020. CA: A Cancer Journal for Clinicians. 2020; 70: 7-30.

[4] Reis FJC. Rastreamento e diagnóstico das neoplasias de ovário papel dos marcadores tumorais. Revista Brasileira de Ginecologia e Obstetrícia. 2005; 27: 222-227.

[5] Reid BM, Permuth JB, Sellers TA. Epidemiology of ovarian cancer: a review. Cancer Biology \& Medicine. 2017; 14: 9-32.

[6] Holschneider CH, Berek JS. Ovarian cancer: epidemiology, biology, and prognostic factors. Seminars in Surgical Oncology. 2000; 19: 3-10.

[7] Pylväs-Eerola M, Karihtala P, Puistola U. Preoperative sérum 8hydroxydeoxyguanosine is associated with chemoresistance and is a powerful prognostic factor in endometrioid-type epithelial ovarian cancer. BMC Cancer. 2015; 15: 493.

[8] Webb PM, Jordan SJ. Epidemiology of epithelial ovarian cancer. Best Practice \& Research. Clinical Obstetrics \& Gynaecology. 2017; 41: 3-14.

[9] Brewer MA, Johnson K, Follen M, Gershenson D, Bast R. Prevention of ovarian cancer: intraepithelial neoplasia. Clinical Cancer Research. 2003; 9: 20-30.

[10] Wu X, Groves FD, McLaughlin CC, Jemal A, Martin J, Chen VW. Cancer incidence patterns among adolescents and young adults in the United States. Cancer Causes \& Control. 2005; 16: 309-320.

[11] Ingersoll SB, Patel S, Caballero L, Ahmad S, Edwards D, Holloway RW, et al. Synergistic cytotoxicity of interferonalpha-2b and interleukin-2 in combination with PBMC against ovarian cancer: development of an experimental model for cellular therapy. Gynecologic Oncology. 2009; 112: 192-198.

[12] Agarwal R, Kaye SB. Prognostic factors in ovarian cancer: how close are we to a complete picture? Annals of Oncology. 2005; 16: 4-6.

[13] Gadducci A, Cosio S, Tana R, Genazzani AR. Serum and tissue biomarkers as predictive and prognostic variables in epithe- 
lial ovarian cancer. Critical Reviews in Oncology/Hematology. 2009; 69: 12-27.

[14] Hirashima K, Watanabe M, Shigaki H, Imamura Y, Ida S, Iwatsuki M, et al. Prognostic significance of the modified Glasgow prognostic score in elderly patients with gastric cancer. Journal of Gastroenterology. 2014; 49: 1040-1046.

[15] Athanassiadou P, Grapsa D, Athanassiades P, Gonidi M, Athanassiadou A, Tsipis A, et al. The prognostic significance of COX-2 and survivin expression in ovarian cancer. Pathology, Research and Practice. 2008; 204: 241-249.

[16] Yang W, Lu Z, Bast RC. The role of biomarkers in the management of epithelial ovarian cancer. Expert Review of Molecular Diagnostics. 2017; 17: 577-591.

[17] Muinao T, Deka Boruah HP, Pal M. Diagnostic and Prognostic Biomarkers in ovarian cancer and the potential roles of cancer stem cells - an updated review. Experimental Cell Research. 2018; 362: 1-10.

[18] Giampaolino P, Foreste V, Della Corte L, Di Filippo C, Iorio $\mathrm{G}$, Bifulco G. Role of biomarkers for early detection of ovarian cancer recurrence. Gland Surgery. 2020; 9: 1102-1111.

[19] Bishara S, Griffin M, Cargill A, Bali A, Gore ME, Kaye SB, et al. Pre-treatment white blood cell subtypes as prognostic indicators in ovarian cancer. European Journal of Obstetrics, Gynecology, and Reproductive Biology. 2008; 138: 71-75.

[20] Templeton AJ, McNamara MG, Šeruga B, Vera-Badillo FE, Aneja P, Ocaña A, et al. Prognostic role of neutrophil-tolymphocyte ratio in solid tumors: a systematic review and metaanalysis. Journal of the National Cancer Institute. 2014; 106: dju124.

[21] Sanguinete MMM, Oliveira PHD, Martins-Filho A, Micheli DC, Tavares-Murta BM, Murta EFC, et al. Serum IL-6 and IL-8 Correlate with Prognostic Factors in Ovarian Cancer. Immunological Investigations. 2017; 46: 677-688.

[22] Martins-Filho A, Jammal MP, Micheli DC, Tavares-Murta BM, Etchebehere RM, Murta EFC, et al. Role of Intracystic Cytokines and Nitric Oxide in Ovarian Neoplasms. Scandinavian Journal of Immunology. 2017; 86: 462-470.

[23] Jammal MP, Martins-Filho A, Silveira TP, Murta EFC, Nomelini RS. Cytokines and Prognostic Factors in Epithelial Ovarian Cancer. Clinical Medicine Insights: Oncology. 2016; 10: CMO.S38333.

[24] Jia L, Ren J, Wang Y, Zheng Y, Zhang H, Zhang Q, et al. Inhibitory role of prohibitin in human ovarian epithelial cancer. International Journal of Clinical and Experimental Pathology. 2014; 7: 2247-2255.

[25] Bamias A, Tsiatas ML, Kafantari E, Liakou C, Rodolakis A, Voulgaris Z, et al. Significant differences of lymphocytes isolated from ascites of patients with ovarian cancer compared to blood and tumor lymphocytes. Association of CD3+CD56+ cells with platinum resistance. Gynecologic Oncology. 2007; 106: 75-81.

[26] Kisielewski R, Tołwińska A, Mazurek A, Laudański P. Inflammation and ovarian cancer - current views. Ginekologia Polska. 2013; 84: 293-297.

[27] Freedman RS, Deavers M, Liu J, Wang E. Peritoneal inflammation - a microenvironment for Epithelial Ovarian Cancer (EOC). Journal of Translational Medicine. 2004; 2: 23.

[28] Balkwill F, Mantovani A. Inflammation and cancer: back to Virchow? Lancet. 2001; 357: 539-545.

[29] Mantovani A, Allavena P, Sica A, Balkwill F. Cancer-related inflammation. Nature. 2008; 454: 436-444.

[30] Li J, Fadare O, Xiang L, Kong B, Zheng W. Ovarian serous carcinoma: recent concepts on its origin and carcinogenesis. Journal of Hematology \& Oncology. 2012; 5: 8.

[31] Honn KV, Tang DG, Crissman JD. Platelets and cancer metastasis: a causal relationship? Cancer Metastasis Reviews. 1992;
11: $325-351$.

[32] Stone RL, Nick AM, McNeish IA, Balkwill F, Han HD, Bottsford-Miller J, et al. Paraneoplastic thrombocytosis in ovarian cancer. The New England Journal of Medicine. 2012; 366: 610-618.

[33] Bellati F, Visconti V, Napoletano C, Antonilli M, Frati L, Panici P, et al. Immunology of Gynecologic Neoplasms: Analysis of the Prognostic Significance of the Immune Status. Current Cancer Drug Targets. 2009; 9: 541-565.

[34] Gavalas NG, Karadimou A, Dimopoulos MA, Bamias A. Immune Response in Ovarian Cancer: how is the Immune System Involved in Prognosis and Therapy: Potential for Treatment Utilization. Clinical and Developmental Immunology. 2010; 2010: $1-15$.

[35] Kusuda T, Shigemasa K, Arihiro K, Fujii T, Nagai N, Ohama K. Relative expression levels of Th1 and Th2 cytokine mRNA are independent prognostic factors in patients with ovarian cancer. Oncology Reports. 2005; 13: 1153-1158.

[36] Sykes L, MacIntyre DA, Yap XJ, Teoh TG, Bennett PR. The Th1:th2 dichotomy of pregnancy and preterm labour. Mediators of Inflammation. 2012; 2012: 967629.

[37] Lowe DB, Storkus WJ. Chronic inflammation and immunologic-based constraints in malignant disease. Immunotherapy. 2011; 3: 1265-1274.

[38] Fridman WH, Galon J, Dieu-Nosjean M, Cremer I, Fisson S, Damotte D, et al. Immune infiltration in human cancer: prognostic significance and disease control. Current Topics in Microbiology and Immunology. 2011; 344: 1-24.

[39] Ellyard JI, Simson L, Parish CR. Th2-mediated anti-tumour immunity: friend or foe? Tissue Antigens. 2007; 70: 1-11.

[40] Zhang L, Conejo-Garcia JR, Katsaros D, Gimotty PA, Massobrio M, Regnani G, et al. Intratumoral $\mathrm{T}$ cells, recurrence, and survival in epithelial ovarian cancer. The New England Journal of Medicine. 2003; 348: 203-213.

[41] Gonzalez H, Hagerling C, Werb Z. Roles of the immune system in cancer: from tumor initiation to metastatic progression. Genes \& Development. 2018; 32: 1267-1284.

[42] Sato E, Olson SH, Ahn J, Bundy B, Nishikawa H, Qian F, et al. Intraepithelial CD8+ tumor-infiltrating lymphocytes and a high CD8+/regulatory $\mathrm{T}$ cell ratio are associated with favorable prognosis in ovarian cancer. Proceedings of the National Academy of Sciences of the United States of America. 2005; 102: 1853818543.

[43] Hagemann T, Wilson J, Burke F, Kulbe H, Li NF, Plüddemann A, et al. Ovarian cancer cells polarize macrophages toward a tumor-associated phenotype. Journal of Immunology. 2006; 176: 5023-5032.

[44] Vakkila J, Lotze MT. Inflammation and necrosis promote tumour growth. Nature Reviews. Immunology. 2004; 4: 641-648.

[45] Galon J. Type, Density, and Location of Immune Cells within Human Colorectal Tumors Predict Clinical Outcome. Science. 2006; 313: 1960-1964.

[46] Santoiemma PP, Powell DJ. Tumor infiltrating lymphocytes in ovarian cancer. Cancer Biology \& Therapy. 2015; 16: 807-820.

[47] Zang RY, Li ZT, Zhang ZY, Cai SM. Surgery and salvage chemotherapy for Chinese women with recurrent advanced epithelial ovarian carcinoma: a retrospective case-control study. International Journal of Gynecological Cancer. 2003; 13: 419427.

[48] Helal TE, Alla AEK, Laban MA, Fahmy RM. Immunophenotyping of tumor-infiltrating mononuclear cells in ovarian carcinoma. Pathology Oncology Research. 2004; 10: 80-84.

[49] Curiel TJ, Coukos G, Zou L, Alvarez X, Cheng P, Mottram P, et al. Specific recruitment of regulatory $\mathrm{T}$ cells in ovarian carcinoma fosters immune privilege and predicts reduced survival. Nature Medicine. 2004; 10: 942-949. 
[50] Lamkin DM, Lutgendorf SK, McGinn S, Dao M, Maiseri H, DeGeest K, et al. Positive psychosocial factors and NKT cells in ovarian cancer patients. Brain, Behavior, and Immunity. 2008; 22: $65-73$.

[51] Santin AD, Bellone S, Ravaggi A, Roman J, Smith CV, Pecorelli $\mathrm{S}$, et al. Increased levels of interleukin-10 and transforming growth factor-beta in the plasma and ascitic fluid of patients with advanced ovarian cancer. BJOG: An International Journal of Obstetrics and Gynaecology. 2001; 108: 804-808.

[52] Shi F, Van Kaer L. Reciprocal regulation between natural killer cells and autoreactive T cells. Nature Reviews. Immunology. 2006; 6: 751-760.

[53] Belisle JA, Gubbels JAA, Raphael CA, Migneault M, Rancourt $\mathrm{C}$, Connor JP, et al. Peritoneal natural killer cells from epithelial ovarian cancer patients show an altered phenotype and bind to the tumour marker MUC16 (CA125). Immunology. 2007; 122: 418-429.

[54] Mantovani A, Bottazzi B, Colotta F, Sozzani S, Ruco L. The origin and function of tumor-associated macrophages. Immunology Today. 1992; 13: 265-270.

[55] Feng W, Dean DC, Hornicek FJ, Shi H, Duan Z. Exosomes promote pre-metastatic niche formation in ovarian cancer. Molecular Cancer. 2019; 18: 124.

[56] Bingle L, Brown NJ, Lewis CE. The role of tumour-associated macrophages in tumour progression: implications for new anticancer therapies. The Journal of Pathology. 2002; 196: 254-265.

[57] Zhang M, He Y, Sun X, Li Q, Wang W, Zhao A, et al. A high M1/M2 ratio of tumor-associated macrophages is associated with extended survival in ovarian cancer patients. Journal of Ovarian Research. 2014; 7: 19.

[58] Kelly-Goss MR, Sweat RS, Stapor PC, Peirce SM, Murfee WL. Targeting Pericytes for Angiogenic Therapies. Microcirculation. 2014; 21: 345-357.

[59] Cirri P, Chiarugi P. Cancer associated fibroblasts: the dark side of the coin. American Journal of Cancer Research. 2011; 1: 482497.

[60] Da Silva AC, Jammal MP, Crispim PCA, Murta EFC, Nomelini RS. The Role of Stroma in Ovarian Cancer. Immunological Investigations. 2020; 49: 406-424.

[61] Martin Lluesma S, Wolfer A, Harari A, Kandalaft LE. Cancer Vaccines in Ovarian Cancer: how can we Improve? Biomedicines. 2016; 4: 10.

[62] Schuster M, Nechansky A, Kircheis R. Cancer immunotherapy. Biotechnology Journal. 2006; 1: 138-147.

[63] Le DT, Brockstedt DG, Nir-Paz R, Hampl J, Mathur S, Nemunaitis J, et al. A live-attenuated Listeria vaccine (ANZ-100) and a live-attenuated Listeria vaccine expressing mesothelin (CRS207) for advanced cancers: phase $i$ studies of safety and immune induction. Clinical Cancer Research. 2012; 18: 858-868.

[64] Odunsi K, Matsuzaki J, Karbach J, Neumann A, MhawechFauceglia P, Miller A, et al. Efficacy of vaccination with recombinant vaccinia and fowlpox vectors expressing NY-ESO-1 antigen in ovarian cancer and melanoma patients. Proceedings of the National Academy of Sciences. 2012; 109: 5797-5802.

[65] Rahma OE, Ashtar E, Czystowska M, Szajnik ME, Wieckowski $\mathrm{E}$, Bernstein $\mathrm{S}$, et al. A gynecologic oncology group phase II trial of two p53 peptide vaccine approaches: subcutaneous injection and intravenous pulsed dendritic cells in high recurrence risk ovarian cancer patients. Cancer Immunology, Immunotherapy. 2012; 61: 373-384.

[66] Dohi S, Ohno S, Ohno Y, Takakura M, Kyo S, Soma G, et al. WT1 peptide vaccine stabilized intractable ovarian cancer patient for one year: a case report. Anticancer Research. 2011; 31: $2441-2445$.
[67] Zhao X, Wei YQ, Peng ZL. Induction of T cell responses against autologous ovarian tumors with whole tumor cell lysate-pulsed dendritic cells. Immunological Investigations. 2001; 30: 33-45.

[68] Schwab CL, English DP, Roque DM, Pasternak M, Santin AD Past, present and future targets for immunotherapy in ovarian cancer. Immunotherapy. 2014; 6: 1279-1293.

[69] Pardoll DM. The blockade of immune checkpoints in cancer immunotherapy. Nature Reviews Cancer. 2012; 12: 252-264.

[70] Nirschl CJ, Drake CG. Molecular pathways: coexpression of immune checkpoint molecules: signaling pathways and implications for cancer immunotherapy. Clinical Cancer Research. 2013; 19: 4917-4924.

[71] Postow MA, Callahan MK, Wolchok JD. Immune Checkpoint Blockade in Cancer Therapy. Journal of Clinical Oncology. 2015; 33: 1974-1982.

[72] Shrikant P, Khoruts A, Mescher MF. CTLA-4 blockade reverses CD8 + T cell tolerance to tumor by a CD4+ T cell- and IL-2dependent mechanism. Immunity. 1999; 11: 483-493.

[73] Dong H, Strome SE, Salomao DR, Tamura H, Hirano F, Flies $\mathrm{DB}$, et al. Tumor-associated B7-H1 promotes T-cell apoptosis: a potential mechanism of immune evasion. Nature Medicine. 2002; 8: 793-800.

[74] Hui E, Cheung J, Zhu J, Su X, Taylor MJ, Wallweber HA, et al. $\mathrm{T}$ cell costimulatory receptor $\mathrm{CD} 28$ is a primary target for PD-1-mediated inhibition. Science. 2017; 355: 1428-1433.

[75] Zou W, Wolchok JD, Chen L. PD-L1 (B7-H1) and PD-1 pathway blockade for cancer therapy: Mechanisms, response biomarkers, and combinations. Science Translational Medicine. 2016; 8: 328rv4.

[76] Ribas A, Shin DS, Zaretsky J, Frederiksen J, Cornish A, Avramis E, et al. PD-1 Blockade Expands Intratumoral Memory T Cells. Cancer Immunology Research. 2016; 4: 194-203.

[77] Wang W, Liu JR, Zou W. Immunotherapy in Ovarian Cancer. Surgical Oncology Clinics of North America. 2019; 28: 447464.

[78] Quirk SK, Shure AK, Agrawal DK. Immune-mediated adverse events of anticytotoxic T lymphocyte-associated antigen 4 antibody therapy in metastatic melanoma. Translational Research. 2015; 166: 412-424.

[79] Mellman I, Coukos G, Dranoff G. Cancer immunotherapy comes of age. Nature. 2011; 480: 480-489.

[80] Della Corte L, Foreste V, Di Filippo C, Giampaolino P, Bifulco G. Poly (ADP-ribose) polymerase (PARP) as target for the treatment of epithelial ovarian cancer: what to know. Expert Opinion on Investigational Drugs. 2021; 30: 543-554.

[81] Lampert EJ, Zimmer A, Padget M, Cimino-Mathews A, Nair JR, Liu Y, et al. Combination of PARP Inhibitor Olaparib, and PDL1 Inhibitor Durvalumab, in Recurrent Ovarian Cancer: a Proofof-Concept Phase II Study. Clinical Cancer Research. 2020; 26: 4268-4279.

[82] Ott PA, Hodi FS, Buchbinder EI. Inhibition of Immune Checkpoints and Vascular Endothelial Growth Factor as Combination Therapy for Metastatic Melanoma: An Overview of Rationale, Preclinical Evidence, and Initial Clinical Data. Frontiers in Oncology. 2015; 5: 202.

[83] Galluzzi L, Zitvogel L, Kroemer G. Immunological Mechanisms Underneath the Efficacy of Cancer Therapy. Cancer Immunology Research. 2016; 4: 895-902.

[84] Langer CJ, Gadgeel SM, Borghaei H, Papadimitrakopoulou VA, Patnaik A, Powell SF, et al. KEYNOTE-021 investigators. Carboplatin and pemetrexed with or without pembrolizumab for advanced, non-squamous non-small-cell lung cancer: a randomised, phase 2 cohort of the open-label KEYNOTE-021 study. Lancet Oncology. 2016; 17: 1497-1508. 